\title{
TRISTAN and Dyno, two laboratory searches for keV sterile neutrinos Dark Matter
}

\section{Thierry Lasserre*t}

Commissariat à l'énergie atomique et aux énergies alternatives, Centre de Saclay, DRF/IRFU, 91191 Gif-sur-Yvette, France

Institute for Advanced Study, Technische Universität München, James-Franck-Str. 1, 85748

Garching

AstroParticule et Cosmologie, Université Paris Diderot, CNRS/IN2P3, CEA/IRFU, Observatoire de Paris, Sorbonne Paris Cité, 75205 Paris Cedex 13, France

E-mail: thierry.lasserre@cea.fr

Two laboratory-based approaches to search for keV sterile neutrino dark matter are discussed: 1) TRISTAN, using the production of sterile neutrinos in the tritium radioactive beta decay and their detection via kinematic considerations, and 2) Dyno, a direct detection of the dark matter particle present in our galaxy through neutrino capture on stable ${ }^{163} \mathrm{Dy}$.

Neutrino Oscillation Workshop

4 - 11 September, 2016

Otranto (Lecce, Italy)

\footnotetext{
* Speaker.

$\dagger$ Thierry Lasserre would like to acknowledge the support of the Technische Universität München Institute for Advanced Study, funded by the German Excellence Initiative and the European Union Seventh Framework Programme under grant agreement No. 291763, as well as the European Union Marie Curie COFUND program.
} 


\section{Introduction}

Although the existence of dark matter (DM) is strongly supported by cosmological observations its nature is unknown. The Standard Model (SM) of elementary particle physics does not provide any suitable option. Hence other candidates have been proposed in several theories beyond the SM. Sterile neutrinos appear in several extensions of the SM as right-handed neutral fermions acting as singlets under the SM gauge group, not interacting but mixing with active neutrinos. A sterile neutrino with a $\mathrm{keV}$ mass scale could exist and account for a great part of DM [2].

\section{TRISTAN: an upgrade of the KATRIN experiment}

The Karlsruhe Tritium Neutrino (KATRIN) Experiment [1] is designed to measure the mass of the active neutrinos with a sensitivity of $200 \mathrm{meV}(90 \% \mathrm{CL})$. To do so it will measure the tritium $\beta$ decay spectrum close to its endpoint with unprecedented precision. In 2017, the first tritium measurements are scheduled to take place.

The Tritium $\beta$-decay spectrum is given as a superposition of the spectra corresponding to each active neutrino mass eigenstate $m\left(v_{i}\right)$, weighted by its mixing amplitude $\left|U_{e i}\right|$ to the electron flavor. A new neutrino mass eigenstate, in the $\mathrm{keV}$ mass range, would add another decay branch to tritium $\beta$ decay. This new component would have a significantly reduced endpoint, a modified spectral shape, and hence lead to a kink-like distortion of the total tritium $\beta$ decay spectrum that can be written as

$$
\frac{d \Gamma}{d E}=\cos ^{2} \theta_{e 4} \frac{d \Gamma}{d E}\left(m\left(v_{\mathrm{e}}\right)\right)+\sin ^{2} \theta_{e 4} \frac{d \Gamma}{d E}\left(m_{\mathrm{s}}\right),
$$

where $\theta_{e 4}$ describes the active-sterile neutrino mixing, and predominantly determines the size of the effect on the spectral shape. The signature is characterized by the mass $m_{s}$ and mixing angle $\theta_{e 4}$ of the sterile neutrino.

TRISTAN [3] is an extension of KATRIN's measurement interval to the entire tritium $\beta$ decay phase space. It will require significany upgrades of the experimental setup, consisting mainly of a new electron detector. The main advantage of TRISTAN is the use of the KATRIN source strength of $10^{11}$ decays/s. From a purely statistical point of view, this luminosity allows to access mixing angles as small as $\theta_{e 4}=10^{-8}$. However, a number of challenges are related to this new measurement mode. To extend the measurement interval to the entire tritium beta decay spectrum a new detector and read-out system has to be designed and realized. An energy resolving Si-based detector system is envisioned, with energy resolution of $300 \mathrm{eV}$ (FWHM). Furthermore, a number of systematic uncertainties become relevant when considering the entire tritium spectrum. These include: 1) Uncertainties in the theoretical description of the spectrum, 2) multiple-scattering of electrons in the source, 3) magnetic trapping of electrons in local magnetic field minima, 4) non-adiabatic motion in the KATRIN main spectrometer, 5) energy loss in the dead-layer of the Si-detector, 5) backscattering on the Si-detector and rear-wall surface, 6) non-linearities in the read-out system. To mitigate the systematic effects, a combination of integral (KATRIN-like) and differential (energy resolving) measurement is considered. Both modes are prone to different classes of systematic effects and hence provide a powerful tool to reject false-positive signals.

The TRISTAN project comprises two phases: During the commissioning phase of KATRIN, only a small amount of tritium will be used. A resulting counting rate of about 1 Mcps can still 
be handled by the KATRIN detector. By scanning the entire tritium spectrum for about 7 days a statistical sensitivity of about $\theta_{e 4}=10^{-4}(90 \% \mathrm{CL})$ could be reached, which would surpass current laboratory based limits. After KATRIN the second stage is to reach a sensitivity of the order of $\theta_{e 4}=10^{-6}(90 \% \mathrm{CL})$. This can be achieved by running for about 3 years with a source strength of $10^{8} \mathrm{cps}$. Accordingly, the systematic effects need to be reduced to the ppm-level.

\section{Dyno: Direct Detection through Sterile Neutrino Capture on a Stable Target}

The goal of Dyno [4] is to search for keV sterile neutrinos $\left(v_{4}\right)$ clustering in the gravitational potential wells of galactic halos. If neutrinos of mass $\mathrm{m}_{4}$ account for the entire local Dark Matter, $\rho_{\mathrm{DM}} \simeq 0.3 \pm 0.1 \mathrm{GeV} \cdot \mathrm{cm}^{-3}$, then their local number density is given by $\mathrm{n}_{v_{4}} \simeq$ $(300 \pm 100) \cdot 10^{3} / \mathrm{m}_{4}(\mathrm{keV}) \mathrm{cm}^{-3}$, and their averaged velocity is $\mathrm{v}_{v_{4}}=220 \mathrm{~km} / \mathrm{s}$.

Electron (anti)neutrino can be $\beta$-captured on a radioactive nucleus $\mathrm{R}, \stackrel{(-)}{v_{\mathrm{e}}}+\mathrm{R} \rightarrow \mathrm{R}^{\prime}+\mathrm{e}^{ \pm}$. Due to the positive energy balance $Q_{\beta}=M_{a}(R)-M_{a}\left(R^{\prime}\right)>0{ }^{1}$ this exothermic reaction is always allowed independently of the value of the incoming neutrino energy $\mathrm{E}_{v}$. The characteristic signal associated with this process as the emerging electron would create a mono-energetic peak at $\mathrm{T}_{\mathrm{e}}=$ $\mathrm{E}_{0}+\mathrm{m}_{v}$, where $\mathrm{T}_{\mathrm{e}}$ is the electron kinetic energy, $\mathrm{E}_{0}$ is the endpoint energy of the $\beta$-decay, and $\mathrm{m}_{v}$ is the effective electron neutrino mass. The detection of the $\mathrm{keV}$ neutrino DM through $v$-capture on $\beta$-decaying nuclei can thus be considered. $\mathrm{keV}$ neutrinos induced mono-energetic electron signal would appear at $\mathrm{T}_{\mathrm{e}}=\mathrm{E}_{0}+\mathrm{m}_{4}$, thus comfortably distinguishable with standard nuclear physics technology. However the expected capture rate suffers from a potentially strong suppression factor $\sin ^{2} \theta_{e 4}$ induced by the mixing between the sterile and active neutrino components. A mass of radioactive material, on the scale of $100 \mathrm{~g}$ for tritium, $10 \mathrm{~kg}$ for ${ }^{106} \mathrm{Ru}$, and 600 tons for ${ }^{163} \mathrm{Ho}$, would thus be required to probe a mixing angle of the order of $\sin ^{2} \theta_{e 4} \sim 10^{-6}$. While procuring this amount of radioactive material is extremely challenging such a mass is in contrast conceivable with a non-radioactive target material.

Indeed keV neutrinos could be capture on a stable nucleus $S$ leading to the radioactive daughter nucleus $D$, produced as a positive ion:

$$
v+{ }_{Z} \mathrm{~S} \rightarrow \mathrm{e}^{-}+{ }_{(\mathrm{Z}+1)} \mathrm{D}^{+} .
$$

This reaction can be stimulated by the mass of a hypothetical sterile neutrino if

$$
\mathrm{m}_{4} \geq \Delta(\mathrm{S})-\Delta(\mathrm{D})-\mathrm{E}_{\mathrm{b}}\left(\mathrm{D}^{+}\right)+\mathrm{E}_{\mathrm{b}}(\mathrm{D}) \simeq \mathrm{Q}_{\beta}^{\mathrm{tab}}
$$

where $E_{b}$ define the binding energies of the orbital electrons. $\Delta$ are the usual tabulated mass excess and $\mathrm{Q}_{\beta}^{\mathrm{tab}}$ denotes the tabulated $\mathrm{Q}$-value assuming $\mathrm{m}_{v}=0$. Thus the $\mathrm{keV}$ sterile neutrino capture on stable nuclei can be stimulated by the neutrino mass energy for $-\mathrm{m}_{v_{4}}<\mathrm{Q}_{\beta}^{\text {tab }} \leq 0$.

The highest negative $\mathrm{Q}_{\beta}^{\mathrm{tab}}$ value is given for ${ }^{163} \mathrm{Dy}$ that mainly captures neutrinos from its nuclear ground state to the ground state of ${ }^{163} \mathrm{Ho}$

$$
\begin{aligned}
{ }^{163} \operatorname{Dy}\left(\mathrm{gs}, \mathrm{I}^{\pi}\right. & \left.=5 / 2^{-}\right)+v_{4}\left(\mathrm{~m}_{4}>2.83 \mathrm{keV}\right) \\
& \rightarrow{ }^{163} \mathrm{Ho}\left(\mathrm{gs}, \mathrm{I}^{\prime \pi^{\prime}}=7 / 2^{-}\right)+e^{-} .
\end{aligned}
$$

\footnotetext{
${ }^{1} \mathrm{M}_{\mathrm{a}}$ denotes the masses of neutral atoms, $\mathrm{M}_{\mathrm{n}}$ denotes the nuclear masses.
} 
Subsequently the ${ }^{163}$ Ho nuclei decays through electron capture (EC) with a half-life of 4570 years. The production rate of ${ }^{163} \mathrm{Ho}$ is given by

$$
\mathrm{R}_{163 \mathrm{Ho}}=\mathrm{N}_{{ }_{163} \mathrm{Dy}} \cdot<\sigma_{\mathrm{c}} \mathrm{v}_{v_{4}}>\cdot \mathrm{n}_{v_{4}} \times \sin ^{2} \theta_{\mathrm{e} 4}
$$

where $N_{163}$ Dy is the number of target ${ }^{163}$ Dy atoms and $\left\langle\sigma_{c} v_{v_{4}}\right\rangle$ denotes the averaged product of the capture cross section and the keV neutrino velocity. In the non-relativistic approximation the cross section can be expressed as

$$
\sigma_{c}\left(\mathrm{~m}_{4}\right) \simeq 4.8 \cdot 10^{-43} \cdot \mathrm{E}_{\mathrm{e}}\left(\mathrm{m}_{4}\right) \cdot \mathrm{p}_{\mathrm{e}}\left(\mathrm{m}_{4}\right) \cdot \mathrm{F}\left(\mathrm{m}_{4}\right) \mathrm{cm}^{2},
$$

where the nuclear size and electron screening effects are taken into account in the Fermi function. It is appealing to consider an integral experiment where the ${ }^{163}$ Dy has been exposed over a geologic time, $t$, enhancing the number of captures given by

$$
\begin{aligned}
& N_{163} \mathrm{Ho}\left(\mathrm{t}, \mathrm{m}_{4}, \sin ^{2} \theta_{\mathrm{e} 4}, \mathrm{~m}_{163}{ }^{15 y}\right)=\frac{\mathrm{m}_{163} 3_{\mathrm{Dy}} \cdot N_{\mathrm{A}}}{\mathrm{A}_{163}{ }_{\mathrm{Dy}} \cdot \lambda_{163}^{\mathrm{EC}} \mathrm{Ho}} \times \\
& \sigma\left(\mathrm{m}_{4}\right)<\mathrm{v}_{v_{4}}>\mathrm{n}_{v_{4}}\left(1-\mathrm{e}^{-\lambda_{163}^{\mathrm{EC}} \mathrm{Ho}} \cdot{ }^{\mathrm{t}}\right) \times \sin ^{2} \theta_{\mathrm{e} 4},
\end{aligned}
$$

where $A_{163}$ Dy is the ${ }^{163}$ Dy molar mass and $m_{163}$ Dy is the target mass. After an exposure of more than 30000 years an equilibrium is reached between the ${ }^{163}$ Ho production and subsequent EC decays. For $m_{4}=5 \mathrm{keV}$ about $7 \cdot 10^{9} \times \sin ^{2} \theta_{e 4}$ atoms of ${ }^{163} \mathrm{Ho}$ are expected in 1 ton of ${ }^{163} \mathrm{Dy}$.

Solar neutrinos $(\mathrm{S} v \mathrm{~B})$ are also captured on ${ }^{163} \mathrm{Dy}$ and thus constitute a background for the DM search. In a 1 ton ${ }^{163}$ Dy target exposed for more than 30000 years $\sim 2.7 \cdot 10^{5}{ }^{163}$ Ho atoms are expected.

The Dyno detection method consists in counting the number of ${ }^{163} \mathrm{Ho}$ atoms in rare-earth ores resulting from the $v$-capture on ${ }^{163} \mathrm{Dy}$. After chemical treatment the atoms could be precisely counted in a magneto-optical trap. A sensitivity of $\sin ^{2} \theta_{e 4} \sim 10^{-5}$ is reachable with a kg-scale target mass of ${ }^{163}$ Dy. Mineral ore containing dysprosium may contain traces of natural uranium and thorium, leading to natural production of ${ }^{163} \mathrm{Ho}$. But this pollution could be minimized to an acceptable level through a careful rock selection, such as the Adamsite. This integral approach is thus limited by the solar neutrino background. Indeed, for $\sin ^{2} \theta_{e 4} \sim 5 \cdot 10^{-5}$, a similar number of ${ }^{163} \mathrm{Ho}$ atoms is produced by both the sterile and solar neutrinos.

Assuming a one percent uncertainty in the knowledge of the solar neutrinos and the atom counting efficiency the sensitivity could asymptotically tend to $\sin ^{2} \theta_{e 4} \sim 10^{-6}$.

\section{References}

[1] J. Angrik et. al., KATRIN design report (2004)

[2] M. Drewes, T. Lasserre, S. Mertens, A. Merle et. al., arXiv:1602.04816 (2016)

[3] S. Mertens et. al., JCAP 1502, 20 (2015)

[4] T. Lasserre et. al., arXiv:1609.04671v2 (2016) 